'Servicio de Urgencias. Parc Taul' Sabadell, Hospital Universitari. Barcelona. Universitat Autònoma de Barcelona. España. ${ }^{2}$ Departamento de Medicina. Universitat Internacional de Catalunya. Barcelona. España. ${ }^{3}$ Unidad de Medical Education. Universitat Internacional de Catalunya. Barcelona. España. ${ }^{4} \mathrm{ABS}$ Manresa-Fundació Althaia. Xarxa Assistencial Universitària. Manresa. Barcelona. España. ${ }^{5}$ ABS Arbúcies Sant Hilari. ICS Girona. España. ${ }^{6} \mathrm{ABS}$ Badia del Vallés. Institut Català de la Salut. Barcelona. España.

?USR Metropolitana Nord, IDIAP Jordi Gol, Cerdanyola, Barcelona, España.

Recibido el 10 de marzo de 2017, aceptado el 17 de enero de 2018.

*La Unidad de Medical Education de Universitat Internacional de Catalunya está formada por:

Àngels Martos, Montserrat Virumbrales, Emili Gené, Esteve Llargués, Lluís Sánchez, Xavier Herranz, Felipe Macias, Beatriz Cabrejas y Aranzazu Balfagon.

Correspondencia a: Dra. Montserrat Virumbrales Cancio

Departament de Medicina. Campus Sant Cugat Universitat Internacional de Catalunya C/Josep Trueta s/n 08195 Sant Cugat del Vallès (Barcelona, España). montsev@uic.es

\title{
Evaluación de competencias en comunicación clínica en estudiantes de medicina con paciente simulado
}

\author{
EMILI GENÉ ${ }^{1,2,3}$, LLUM OLMEDO 2,4, MARTA PASCUAL ${ }^{2,5}$, \\ RAFAEL AZAGRA ${ }^{6,7}$, MARTA ELORDUY $^{2}$, \\ MONTSERRAT VIRUMBRALES 2,3 \\ en nombre de la Unidad de Medical Education*
}

\section{Evaluation of clinical communication skills in medical students with simulated patients}

Background: Communication skills are not learnt innately. It is therefore necessary to both train and evaluate medical students in this area. Aim: To evaluate communication skills of fourth-year medical students with the use of a simulated patient (SP). Material and Methods: Four clinical scenarios (CS) for clinical interviews with simulated patients were designed: chronic and acute diseases, difficult and functional patients. Each student performed the four CS, and his communication skills were evaluated using our own questionnaire as agreed with the SP. The questionnaire included items on verbal and nonverbal communication, warmth, respect, specificity, assertiveness and empathy (rated from 0 to 10). The response variable was the arithmetic mean of the scores on each item. All students received a detailed evaluation report. Results: Sixty one students (32 men) performing 244 interviews, were evaluated. The overall mean score was 7.87 (4.62 to 9.03). The highest scores were for respect and specificity ( 7.57 and 7.15 respectively). The lowest were for empathy and nonverbal communication (6.44 and 6.84 respectively). The CS evaluations were 7.87 (chronic disease), 7.02 (difficult patient), 6.46 (acute disease) and 6.14 (functional pathology). Women had higher overall scores compared to men (7.26 and 6.51 respectively; $p<0.01$ ) and a significantly higher score in all communication variables $(p<0.01)$. Conclusions: The overall assessment in clinical communication with SP is satisfactory although there is room for improvement, especially in empathy and nonverbal communication. Women had significantly higher scores than men.

(Rev Med Chile 2018; 146: 160-167)

Key words: Clinical Competence; Health Communication; Patient Simulation.

esde los inicios de la medicina se ha conferido especial importancia a la relación médico-paciente (RMP) por ser clave para el éxito en la gestión asistencial. La relación asistencial combina la gestión del conocimiento y la aplicación de la tecnología con el factor humano. Esta combinación es fundamental para que la ac- ción del médico pueda responder a las necesidades del enfermo durante la entrevista clínica.

Una buena entrevista clínica es "el marco de interacción humana en la que el paciente y el médico comparten información acerca de uno o varios problemas de salud con el objetivo de llegar a un diagnóstico y tratamiento, $y$, como resultado de 
ello, al establecimiento de la relación de confianza con el paciente y la familia"'.

Por otra parte, la RMP tiene lugar principalmente en la entrevista clínica y, como toda relación que se establece entre dos seres humanos, debe tener como característica principal la confianza. Para que el clima de la entrevista clínica sea de confianza es indispensable tanto la honestidad del paciente como la competencia en comunicación del médico. Así, entre ambas partes, se establecen unos niveles de reciprocidad, que permiten al paciente poder expresar su demanda; $y$ al profesional poder lograr el cumplimiento de las medidas propuestas.

Progresivamente, se ha ido reconociendo la importancia que tiene la comunicación y su influencia en una buena $\mathrm{RMP}^{2,3}$. Hoy en día, se reconoce que si la comunicación clínica es efectiva tendrá un impacto positivo tanto en el diagnóstico como en el cumplimiento de las recomendaciones terapéuticas $^{4-6}$. Merriel et al. demostraron que, si existe una buena RMP, durante la consulta el paciente comunicará un mayor número de problemas y de mayor gravedad ${ }^{4}$. Por otro lado, se ha observado que la formación médica en habilidades comunicativas mejora la relación con el paciente y disminuye el tiempo de consulta ${ }^{7,8}$.

No obstante, desde el punto de vista del paciente y sus familiares, hay una creciente opinión de que el médico "no sabe comunicarse con el paciente" . La sociedad actual espera de los profesionales que, además de conocimientos, habilidades técnicas y dominio en la toma de decisiones, tengan control de las propias emociones, sepan valorar los aspectos emocionales de los pacientes y sobretodo empleen la comunicación de forma efectiva.

Un error frecuente es creer que los años de ejercicio profesional enseñan a realizar una buena entrevista clínica. La experiencia puede ser fundamental, pero sólo cuando hay una formación previa. Así, la comunicación debe considerase una competencia transversal en la formación de los profesionales de la salud, tan importante como la competencia clínica ${ }^{10}$. Por este motivo, y teniendo en cuenta que la comunicación es una habilidad que se aprende y no una característica innata, es fundamental formar y evaluar en habilidades comunicativas, tanto en el grado de medicina como en la formación médica continuada.

Con la puesta en marcha del Espacio Europeo de Educación Superior, se han ido incluyendo en los planes de estudio de medicina, aunque de forma no homogénea, asignaturas centradas en la adquisición de habilidades comunicativas. En la actualidad, el entorno de simulación clínica con paciente simulado (PS) cobra fuerza para la adquisición de estas competencias en comunicación y entrevista clínica. El encuentro de los estudiantes con el PS supone un aprendizaje de contacto directo, que les permite hacer, reconocer y corregir errores, especialmente cuando la interacción va seguida de un "debriefing" o retroalimentación docente y de una autorreflexión que facilite la continuación de su proceso de autoaprendizaje $\mathrm{e}^{11-13}$.

No obstante, la evaluación formativa de habilidades comunicativas sigue presentando actualmente, ciertas debilidades relacionadas con las estrategias, el tipo y los instrumentos empleados ${ }^{14-16}$.

En este contexto, el objetivo de este estudio es evaluar las habilidades comunicativas de estudiantes de cuarto curso de medicina con PS, mediante la aplicación de un cuestionario específico, a fin de identificar fortalezas y debilidades y proponer aspectos de mejora.

\section{Material y Método}

Estudio observacional y transversal realizado con estudiantes de cuarto curso de medicina de la Universitat Internacional de Catalunya.

En el marco de la asignatura de Medicina Familiar y Comunitaria, se diseñó una actividad sobre comunicación médico-paciente con PS, obligatoria, formativa-evaluativa pero sin impacto en la calificación final de la asignatura. Esta actividad se realizó en abril de 2014, dividiendo a los 64 estudiantes matriculados en cuatro grupos. Cada grupo estaba formado por 16 estudiantes. Cada uno de los cuatro grupos realizó esta actividad durante 4 h: 10 min de entrevista clínica y cinco minutos para el cambio de escenario (Figura 1).

El responsable de la asignatura y los profesores de la misma diseñaron cuatro escenarios clínicos (EC) en base a trabajar la comunicación en las situaciones clínicas de mayor interés en Atención Primaria (AP): EC1) patología crónica prevalente en AP, EC2) patología aguda prevalente en AP, EC3) patología funcional (entrevista clínica realizada con PS y acompañante) y EC4) paciente difícil. En cada EC se diseñaron cuatro casos clínicos 
diferentes para evitar el traspaso de información entre los estudiantes, teniendo en cuenta que la competencia a trabajar era comunicación y no otras competencias como anamnesis, exploración física o manejo clínico. Todos los EC podían resolverse desde AP sin necesidad de derivación del paciente a la atención especializada.

Cada estudiante realizó los cuatro EC y fue grabado durante el desarrollo de uno de ellos,

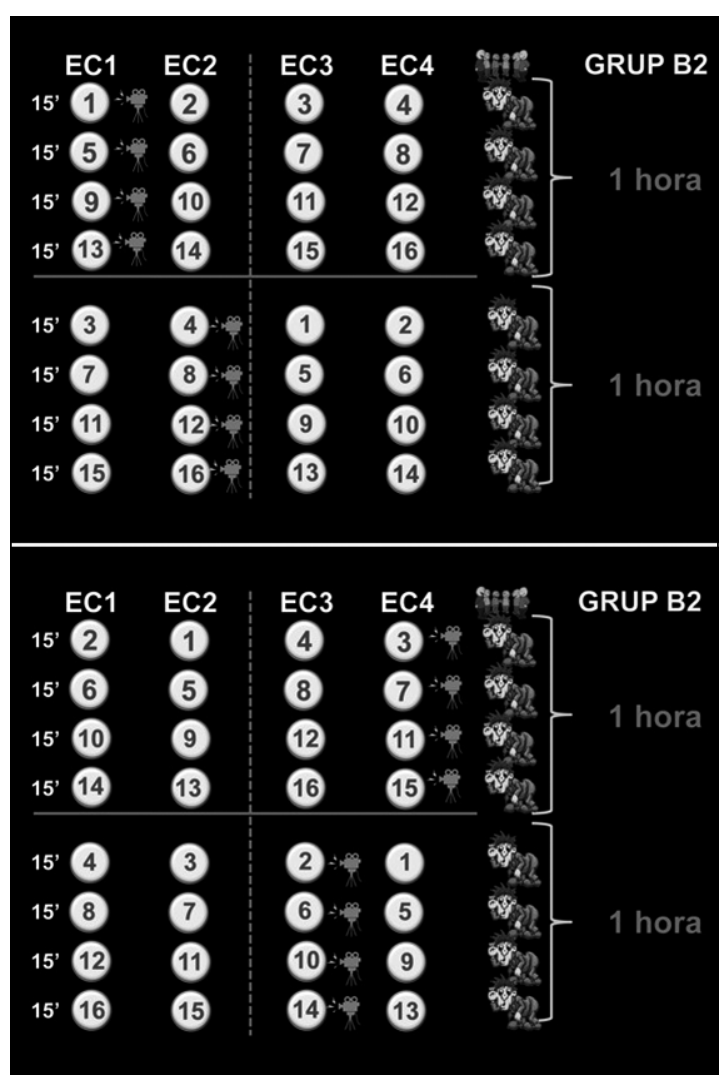

Figura 1. Organización de las sesiones. Los estudiantes se dividieron en cuatro grupos de 16 estudiantes. Cada sesión tenía una duración de 4 h: 10 min para la entrevista clínica y 5 min para el cambio de estación clínica durante los que el paciente simulado realizaba la evaluación del estudiante. Todos los estudiantes tenían el esquema de esta figura. Cada estudiante tenía asignado un número para saber qué escenario clínico debía realizar. La cámara indica el escenario clínico en que se realizaba la grabación. Durante la realización de los cuatro escenarios clínicos (cuatro alumnos por ronda), el resto del grupo (doce alumnos) esperaba en un aula de la Facultad. De esta manera cada alumno realiza los cuatro escenarios clínicos y dispone de una entrevista clínica con videograbación. EC1: Patología crónica prevalente en Atención Primaria; EC2: Patología aguda prevalente en Atención Primaria; EC3: Patología funcional EC4: Paciente difícil. de manera rotatoria y aleatoria. Todos firmaron su consentimiento una vez informados de que el propósito de las grabaciones era únicamente académico.

Las variables registradas fueron: demográficas (edad, sexo); de comunicación (verbal, no verbal, calidez, respeto, concreción, asertividad y empatía) y otras relacionadas con la práctica clínica (uso de tecnicismos y derivación del PS a la atención especializada), todas ellas valoradas mediante escala tipo Likert de 0 a 10. La herramienta de evaluación fue la "comunicación global" (variable respuesta) calculada como la media aritmética de las siete variables de comunicación registradas. También se analizaron los valores obtenidos en cada una de las variables de comunicación que comportan aspectos cualitativos de la relación asistencial.

La evaluación de los alumnos fue realizada por los PS. Los PS eran profesionales sanitarios, todos ellos formados, durante los dos años previos, en metodología para la interpretación y evaluación de escenarios clínicos (EC). El PS disponía de cinco minutos, entre cada escenario, para evaluar al estudiante en base a su percepción como paciente de las diferentes variables de comunicación registradas. La valoración se realizaba mediante dispositivo portátil en el que se recogían las diferentes variables (cuestionario) y se volcaban los datos a una hoja de cálculo (Microsoft Excel ${ }^{\oplus}$ ).

El análisis estadístico se realizó con el paquete estadístico SPPS 18.0. Las variables cuantitativas se describen mediante la media y la desviación estándar, y las variables cualitativas mediante porcentajes. Las características basales cuantitativas se comparan con la prueba t de Student o U de Mann-Whitney según sigan o no una distribución normal. Se acepta como nivel de significación estadística un valor de $\mathrm{p}<0,05$.

Los procedimientos utilizados respetaron las normas éticas concordantes con la Declaración de Helsinki.

\section{Resultados}

De los 64 estudiantes matriculados, 61 (95,3\%) realizaron la actividad. Se evaluaron un total de 244 entrevistas y se realizaron 61 grabaciones, una por estudiante. El 52,4\% de los estudiantes fueron hombres.

La nota media obtenida en "comunicación 
global" fue 6,87 (4,62-9,03). Cuatro presentaron una puntuación inferior a cinco. La distribución de los estudiantes en función de la nota obtenida puede verse en la Figura 2.

Las variables de comunicación mejor valoradas fueron "respeto" y "concreción" con una puntuación de 7,57 y 7,15 respectivamente. Por el contrario, las variables que obtuvieron una puntuación más baja fueron "comunicación no verbal" y "empatía”, 6,61 y 6,44 respectivamente. La puntuación de cada una de las variables puede verse en la Tabla 1.

Respecto a los cuatro EC, el de patología crónica prevalente fue en el que los estudiantes obtuvieron la mejor valoración en "comunicación global” $(7,87)$, seguido del de paciente difícil $(7,02)$, patología aguda prevalente $(6,46)$ y patología funcional $(6,14)$. Las puntuaciones obtenidas
Figura 2. Distribución de los estudiantes en función de la nota obtenida en comunicación global. La nota de comunicación global es la media aritmética de las siete variables de comunicación evaluadas en la actividad de simulación.

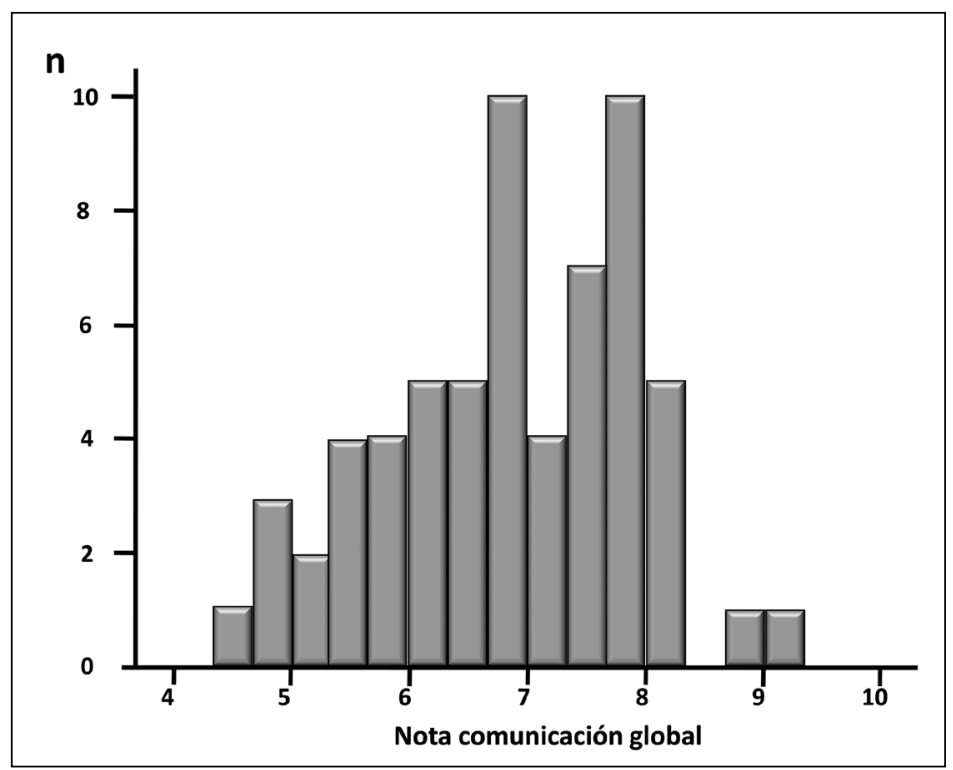

Tabla 1. Puntuación obtenida por los estudiantes en sus entrevistas clínicas con paciente simulado (valoradas de 0 a 10) para las diferentes variables de comunicación y para la variable comunicación global tanto para el conjunto de estudiantes como las puntuaciones en cada uno de los cuatro escenarios clínicos. Los valores se expresan como media y desviación estándar

\begin{tabular}{|lccccc|}
\hline Variables & $\begin{array}{c}\text { Grupo } \\
(\mathbf{n}=\mathbf{2 4 4})\end{array}$ & $\begin{array}{c}\text { EC1 } \\
(\mathbf{n}=\mathbf{6 1})\end{array}$ & $\begin{array}{c}\text { EC2 } \\
(\mathbf{n}=\mathbf{6 1})\end{array}$ & $\begin{array}{c}\text { EC3 } \\
(\mathbf{n}=\mathbf{6 1})\end{array}$ & $\begin{array}{c}\text { EC4 } \\
(\mathbf{n}=\mathbf{6 1})\end{array}$ \\
\hline Comunicación verbal & $6,84 \pm 1,62$ & $7,75 \pm 1,43$ & $6,23 \pm 1,04$ & $6,2 \pm 1,77$ & $7,22 \pm 1,59$ \\
\hline Comunicación no verbal & $6,61 \pm 1,77$ & $7,72 \pm 1,38$ & $6,27 \pm 1,21$ & $6,08 \pm 1,81$ & $6,37 \pm 2,09$ \\
\hline Calidez & $6,70 \pm 1,80$ & $7,74 \pm 1,57$ & $6,48 \pm 1,08$ & $5,92 \pm 1,80$ & $6,7 \pm 2,08$ \\
\hline Respeto & $7,57 \pm 1,70$ & $8,54 \pm 1,47$ & $6,45 \pm 1,12$ & $6,9 \pm 1,23$ & $8,43 \pm 2,04$ \\
\hline Concreción & $7,15 \pm 1,59$ & $7,92 \pm 1,58$ & $6,71 \pm 1,03$ & $6,44 \pm 1,68$ & $7,53 \pm 1,57$ \\
\hline Asertividad & $6,88 \pm 1,91$ & $7,7 \pm 1,63$ & $6,68 \pm 1,30$ & $5,9 \pm 2,08$ & $7,23 \pm 2,08$ \\
\hline Empatía & $6,44 \pm 2,05$ & $7,84 \pm 1,61$ & $6,45 \pm 1,28$ & $5,67 \pm 1,88$ & $5,8 \pm 2,51$ \\
\hline Comunicación global & $6,87 \pm 1,59$ & $7,87 \pm 1,39$ & $6,46 \pm 1,08$ & $6,14 \pm 1,61$ & $7,02 \pm 1,68$ \\
\hline
\end{tabular}

EC1: Patología crónica prevalente en AP; EC2: Patología aguda prevalente en atención primaria; EC3: Patología funcional; EC4: Paciente difícil. 
por cada variable en cada uno de los EC pueden verse en la Tabla 1.

Analizados los resultados por género, las mujeres obtuvieron una puntuación en comunicación global de 7,26 frente a una puntuación de 6,51 de los hombres $(\mathrm{p}<0,0001)$. El porcentaje de mujeres en primer cuartil en función de la puntuación obtenida en "comunicación global" fue de $75 \%$, mientras que en cuarto cuartil fue de $13,4 \%$ (Figura 3 ). Las puntuaciones obtenidas en las diferentes variables de comunicación fueron también superiores en las mujeres (Tabla 2).

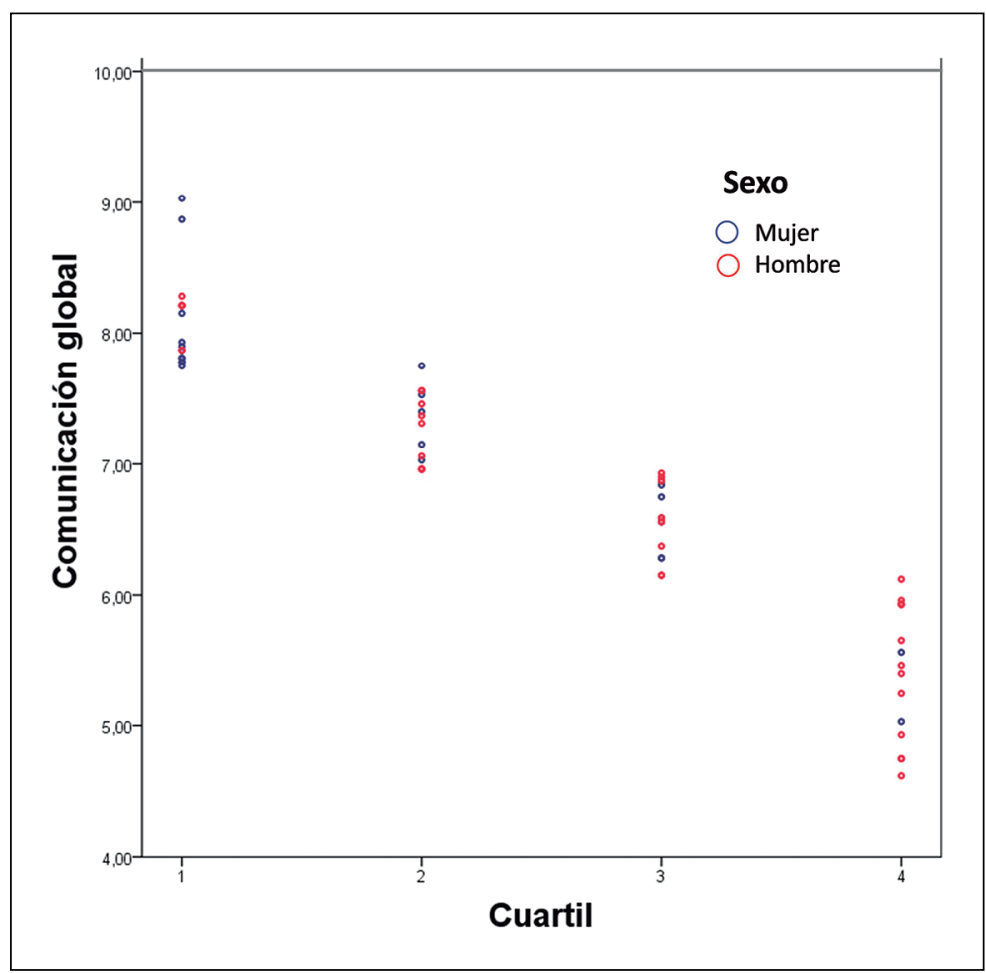

Figura 3. Distribución en función del sexo de los estudiantes respecto a la valoración de comunicación global y el cuartil correspondiente.

\section{Tabla 2. Diferencias en las puntuaciones obtenidas por los estudiantes en sus entrevistas clínicas con paciente simulado (valoradas de 0 a 10) para las diferentes variables de comunicación y para la variable comunicación global por sexos y utilización de grabación o no}

\begin{tabular}{|c|c|c|c|c|c|c|}
\hline \multirow[t]{2}{*}{ Variables } & \multicolumn{3}{|c|}{ Entrevista según sexo } & \multicolumn{3}{|c|}{ Entrevistas según grabación } \\
\hline & $\begin{array}{l}\text { Mujeres } \\
(n=116)\end{array}$ & $\begin{array}{l}\text { Hombres } \\
(n=128)\end{array}$ & p valor & $\begin{array}{c}\text { Sí } \\
(n=61)\end{array}$ & $\begin{array}{c}\text { No } \\
(n=128)\end{array}$ & p valor \\
\hline Comunicación verbal & $7,23 \pm 1,35$ & $6,49 \pm 1,76$ & $<0,0001$ & $7,02 \pm 1,51$ & $6,79 \pm 1,65$ & 0,33 \\
\hline Comunicación no verbal & $7,06 \pm 1,55$ & $6,20 \pm 1,86$ & $<0,0001$ & $6,82 \pm 1,68$ & $6,54 \pm 1,80$ & 0,28 \\
\hline Calidez & $7,13 \pm 1,67$ & $6,31 \pm 1,83$ & $<0,0001$ & $6,80 \pm 1,83$ & $6,67 \pm 1,79$ & 0,61 \\
\hline Respeto & $7,84 \pm 1,52$ & $7,34 \pm 1,82$ & 0,02 & $7,70 \pm 1,49$ & $7,53 \pm 1,76$ & 0,48 \\
\hline Concreción & $7,42 \pm 1,42$ & $6,90 \pm 1,70$ & 0,01 & $7,20 \pm 1,43$ & $7,13 \pm 1,65$ & 0,78 \\
\hline Asertividad & $7,28 \pm 1,63$ & $6,51 \pm 2,08$ & 0,001 & $6,82 \pm 1,81$ & $6,90 \pm 1,95$ & 0,78 \\
\hline Empatía & $6,99 \pm 1,88$ & $5,95 \pm 2,07$ & $<0,0001$ & $6,48 \pm 2,08$ & $2,43 \pm 2,04$ & 0,88 \\
\hline Comunicación global & $7,26 \pm 1,40$ & $6,51 \pm 1,67$ & $<0,0001$ & $6,97 \pm 1,45$ & $6,84 \pm 1,64$ & 0,58 \\
\hline
\end{tabular}


Tabla 3. Uso de tecnicismos y de derivación del paciente simulado a la atención especializada en cada uno de los cuatro escenarios clínicos

\begin{tabular}{|lccccc|}
\hline Variables & $\begin{array}{c}\text { Grupo } \\
(\mathbf{n}=\mathbf{2 4 4})\end{array}$ & $\begin{array}{c}\text { EC1 } \\
(\mathbf{n}=\mathbf{6 1})\end{array}$ & $\begin{array}{c}\text { EC2 } \\
(\mathbf{n}=\mathbf{6 1})\end{array}$ & $\begin{array}{c}\text { EC3 } \\
(\mathbf{n}=\mathbf{6 1})\end{array}$ & $\begin{array}{c}\text { EC4 } \\
(\mathbf{n}=\mathbf{6 1})\end{array}$ \\
\hline Uso de tecnicismos & $39,7 \%$ & $29,5 \%$ & $32,7 \%$ & $39,3 \%$ & $57,3 \%$ \\
\hline Derivación a la atención especializada & $30,3 \%$ & $21,3 \%$ & $13,1 \%$ & $39,3 \%$ & $47,5 \%$ \\
\hline
\end{tabular}

EC1: Patología crónica prevalente en AP; EC2: Patología aguda prevalente en atención primaria; EC3: Patología funcional; EC4: Paciente difícil.

La valoración de la "comunicación global" en aquellos EC donde se realizó grabación fue de 6,97 mientras que en los que no se realizó fue de 6,84 $(\mathrm{p}=0,33)$ (Tabla 2).

Finalmente, el uso de tecnicismos estuvo presente en $39,7 \%$ y el estudiante decidió derivar al PS a la atención especializada en 30,3\% de las entrevistas. El EC del paciente difícil fue en el que más tecnicismos se usaron $(57,3 \%)$ y en el que se derivó más veces $(47,5 \%)$ (Tabla 3$)$.

\section{Discusión}

La puntuación media en comunicación clínica obtenida por los estudiantes de medicina de cuarto curso $(6,87)$, en base a las entevistas clínicas con PS, es esperanzadora teniendo en cuenta que quedan todavía dos años para trabajar esta competencia, durante los que realizarán además el rotatorio clínico. Los aspectos en que han obtenido mejor valoración son el respeto y la concreción, mientras que la comunicación no verbal y la empatía son los aspectos con mayor margen de mejora.

Las puntuaciones obtenidas en los diferentes EC no han sido homogéneas. A priori, el paciente difícil era el escenario más complejo dado que los estudiantes, aún no se habían encontrado con este tipo de pacientes. El paciente difícil genera incomodidad, malestar y rechazo, dificultando estos elementos una comunicación empática. No obstante, este escenario ha sido el segundo mejor puntuado. Esto posiblemente se debe al peso que han tenido las variables de respeto y concreción en la comunicación global. Teniendo en cuenta que sólo desde el respeto es posible articular respuestas empáticas, pensamos que nuestros estudiantes tienen la base para mejorar la empatía, siendo nuestro deber reforzar los aspectos formativos vinculados al control de las emociones. Este punto es de especial interés, pues la principal dificultad atribuible al profesional, que impide ser más empático, es la escasa capacidad de autocontrol ${ }^{17}$.

El EC en que peor valoración han obtenido los estudiantes ha sido el del paciente funcional, posiblemente por las características de estos pacientes (pacientes sin una patología orgánica, en el que su entorno psicosocial juega un papel importante) y por la influencia del acompañante que en ocasiones genera interferencias que dificultan la RMP. La valoración obtenida en los otros dos EC (patología aguda y patología crónica prevalente en AP) manifiesta que los estudiantes han estado más cómodos cuando sus conocimientos clínicos les permiten manejarse con mayor tranquilidad y seguridad.

Los resultados de nuestro estudio reflejan que en la RMP las mujeres comunican mejor que los hombres. Las mayores diferencias (más de un punto) entre géneros se encuentran en la empatía. El comportamiento comunicativo de un varón no es el mismo que el de una mujer ${ }^{18}$; las mujeres poseen un mejor tratamiento de las estructuras lingüísticas, utilizan mejor las pausas y los silencios, y tienen unos movimientos corporales más tenues. Teniendo en cuenta que para efectuar un cambio en los aspectos comunicativos éstos deben abordarse lo antes posible, el poder incidir desde la formación del grado puede ayudar a desarrollar estas capacidades en los estudiantes $\mathrm{y}$, en la medida de lo posible, evitar las diferencias de género ${ }^{19}$.

Es importante resaltar el hecho de que las valoraciones obtenidas por los estudiantes no son peores en los EC en los que se realizó grabación, lo que da validez a su uso ya que no influye en los resultados y sin embargo facilita el "debriefing" posterior y la autorreflexión de los estudiantes. 
Finalmente, destacar que en casi el $40 \%$ de las entrevistas clínicas los estudiantes usaron tecnicismos. Su uso dificulta la comunicación en la RMP y puede ser la causa de una peor valoración. El uso de tecnicismos fue más frecuente en los EC de paciente difícil y funcional. Esto puede deberse a que, en estos, el estudiante tiene menos conocimientos y menor seguridad en sí mismo para manejar la entrevista. Esto mismo también puede justificar que sea en estos EC en los que más se indique la derivación del paciente a atención especializada $(47,5 \%$ y $39,3 \%)$. Los resultados de nuestro estudio ponen en valor el análisis de la competencia de comunicación clínica en un entorno de simulación en AP. No requiere grandes recursos técnicos ni materiales y permite transmitir a cada estudiante los aspectos de mejora en comunicación mediante el "debriefing" y la autorreflexión dirigida. Cada estudiante recibió un informe cuantitativo con sus valoraciones y su posición respecto al grupo de la actividad y respecto al resto de sus compañeros.

El presente estudio presenta algunas limitaciones y fortalezas. Una de las limitaciones podría ser que los resultados no son generalizables y que las diferencias observadas en los diferentes EC fueran atribuibles a diferencias entre observador. No obstante, los evaluadores habían sido formados en la valoración de esta competencia durante los dos años previos y los casos habían sido previamente validados.

Por el contrario, presenta cuatro fortalezas. La primera es que dentro de cada EC se diseñaron diversas situaciones clínicas pra dificultar que los estudiantes pudieran traspasarse la información entre ellos. En segundo lugar, contar con un equipo de PS entrenados durante dos cursos académicos previos a la evaluación de la actividad. La tercera radica en poder utilizar un PS en vez de un paciente estandarizado. El PS tiene capacidad para poder interactuar con el estudiante en función de la respuesta que éste presente frente a las demandas del PS. Este punto es de especial interés en los EC del paciente funcional y difícil. En este último caso, el PS fue instruido en dos papeles, uno más favorecedor y otro en el que persiste en su demanda, según la capacidad del estudiante de empatizar con él. Finalmente, destacar que el hecho de que la actividad sea formativa y evaluativa, pero sin impacto en la nota, pensamos que es una fortaleza dado que el estudiante puede estar pendiente de realizar correctamente la actividad sin preocuparse por la repercusión en la nota final.

Este estudio abre nuevas líneas de investigación en educación médica en el entorno de simulación clínica. Así, queda por conocer si estos resultados se mantienen en futuras promociones o si son promoción-dependientes. Con estos resultados, podemos concluir que la valoración global en comunicación clínica de los estudiantes de cuarto de medicina con PS es satisfactoria, si bien queda un amplio margen de mejora, especialmente en empatía y comunicación no verbal. Las mujeres presentan una puntuación en aspectos comunicativos significativamente más elevada que los hombres.

Agradecimientos: A Vicente Barrioluengo por su paciencia en la grabación y edición de las entrevistas clínicas. A Mercè Fayos, Carme Campos, M. Dolores Ferrer, Araceli González, Josep Antoni Pijuan, Clara Alavedra, Andrés Gadea, Cristina Carod y David Bea, por su paciencia en la interpretación de los PS. A todos los estudiantes que han realizado esta actividad de simulación por su implicación, que ha posibilitado la formación de los PS y la validación de los casos.

\section{Referencias}

1. Borrell F, Prados JA. Entrevista clínica. Conceptos generales. En: Espinás Boquet J. Guía de actuación en Atención Primaria, semFYC, Barcelona, 2011.

2. Ridd MJ, Lewis G, Peters TJ, Salisbury C. Patient-Doctor Depth-of-Relationship Scale: development and validation. Ann Fam Med 2011; 9 (6): 538-45.

3. Ghobadifar MA. Which one is more important to being a good family physician for medical students, disease diagnosis or patient-doctor relationship? Korean J Fam Med 2013; 34 (4): 295-6.

4. Merriel SW, Salisbury C, Metcalfe C, Ridd M. Depth of the patient-doctor relationship and content of general practice consultations: cross-sectional study. Br J Gen Pract 2015; 65 (637): e545-51.

5. Alves de Lima. Habilidades de Comunicación: un pilar básico de la competencia clínica. Revista del CONAREC Disponible en http://www.conarec.org.ar. 2003

6. Cófreces P, Ofman SD, Stefani D. La comunicación en la relación médico-paciente. Análisis de la literatura científica entre 1990 y 2010. Revista de Comunicación y Salud 2014; 4: 19-34. 
7. Langewitz WA, Eich P, Kiss A, Wossmer B. Improving communication skills-a randomized controlled behaviorally oriented intervention study for residents in internal medicine. Psychosomatic Medicine 1998; 60 (3): 268-76.

8. Nystrup J, Larsen J-H, Risør O. World Federation for Medical Education, Faculty of Health Sciences, The Panum Institute, University of Copenhagen, Copenhagen, Denmark. (2010).

9. Clèries X, Borrell F, Epstein RM, Kronfly E, Escoda JJ, Martínez-Carretero JM. Aspectos comunicacionales: el reto de la competencia de la profesión médica. Aten Primaria 2003; 32 (2): 110-7.

10. Clèries X. La esencia de la comunicación en educación médica. Educ Med 2010; 13 (1): 25-31.

11. Ryan CA, Walshe N, Gaffney R, Shanks A, Burgoyne L, Wiskin CM. Using standardized patients to assess communication skills in medical and nursing students. BMC Med Educ 2010; 10: 24.

12. Morrow JB, Dobbie AE, Jenkins C, Long R, Mihalic A, Wagner J. First-year medical students can demonstrate EHR-specific communication skills: a control-group study. Fam Med 2009; 41: 28-33.

13. Valero M, Aramburu J, Baños JE, Sentí M, Pérez J.
Introducción de un portafolio para fomentar competencias transversales de los estudiantes universitarios. Educ Med 2007; 10: 244-51.

14. Salazar-Blanco OF, Echeverri-Restrepo C, Díaz-Hernández DP. Reflexiones sobre la competencia comunicativa en los pregrados de las áreas de salud de universidades colombianas. FEM 2015; 18 (3): 211-7.

15. Nolla-Domenjó M. La evaluación en educación médica. Principios básicos. Educ Med 2009; 12: 223-9.

16. Rider EA, Hinrichs MM, Lown BA. A model for communication skills assessment across de undergraduate curriculum. Med Teach 2006; 28: 27-34.

17. Merayo A, Bravo E, Gordón F. La comunicación con el paciente. Habilidades emocionales para los profesionales de la salud. Barcelona: Elsevier; 2014.

18. Tusón A. Lenguaje, interacción y diferencia sexual. En: González A, Lomas C, coordinadores. Mujer y educación. Educar para la igualdad, educar desde la diferencia. Barcelona: Graó; 2002: 61-76.

19. Encabo Fernández E, López Valero A. Diferencias de género y comunicación: aspectos no verbales y propuestas didácticas. Didáctica (Lengua y Literatura) 2004; 16: 45-56. 